\title{
Numerical study of Fermi-Pasta-Ulam recurrence for water waves over finite depth
}

\author{
V. P. Ruban* \\ Landau Institute for Theoretical Physics, 2 Kosygin Street, 119334 Moscow, Russia
}

(Dated: June 2, 2021)

\begin{abstract}
Highly accurate direct numerical simulations have been performed for two-dimensional free-surface potential flows of an ideal incompressible fluid over a constant depth $h$, in the gravity field $g$. In each numerical experiment, at $t=0$ the free surface profile was in the form $y=A_{0} \cos (2 \pi x / L)$, and the velocity field $\mathbf{v}=0$. The computations demonstrate the phenomenon of Fermi-Pasta-Ulam (FPU) recurrence takes place in such systems for moderate initial wave amplitudes $A_{0} \lesssim 0.12 h$ and spatial periods at least $L \lesssim 120 h$. The time of recurrence $T_{\mathrm{FPU}}$ is well fitted by the formula $T_{\mathrm{FPU}}(g / h)^{1 / 2} \approx 0.16(L / h)^{2}\left(h / \widetilde{A}_{0}\right)^{1 / 2}$.
\end{abstract}

PACS numbers: 47.35.-i, 47.15.K-, 47.10.-g

Many nonlinear dispersive waves are known to exhibit the Fermi-Pasta-Ulam (FPU) recurrence, when a (finitesize) system nearly repeats its initial state after some period of evolution. For the first time, this phenomenon was observed in the famous numerical experiment [1] with one-dimensional (1D) lattices of nonlinear oscillators. The impact of that observation on the subsequent development of nonlinear science was very deep. In particular, it resulted in the discovery of solitons by Zabusky and Kruskal [2]. Since then, the FPU recurrence and related phenomena were studied in many physical contexts (see, e.g., [3 13], and references therein).

In general, the FPU recurrence takes place in a system if its dynamics is nearly integrable. Two different reasons can result in such approximate integrability. In the first case, just a few (2-3) collective degrees of freedom are effectively involved into the evolution due to a small system size and low level of nonlinearity, and that restricted dynamics is integrable (for example, such variant of FPU recurrence takes place for 3D deep-water waves [7, 11]). In the second possible case, the evolution is governed by equations of motion which are close to some completely integrable system [3]. The first case does not require any additional symmetry and therefore is more common, while the second case is more interesting, since it allows for a large number of degrees of freedom to be involved into the process.

As the theory of water waves is concerned, there are three the most popular integrable models for three essentially different dynamic regimes: (i) the nonlinear Schroedinger equation (NLSE) approximates an envelope of deep-water waves, (ii) the Korteweg-de-Vries (KdV) equation describes weakly nonlinear dispersive unidirectional shallow-water waves, and (iii) the Boussinesq equations approximately describe bidirectional shallow-water waves (concerning integrability of the Boussinesq equations, see [14-18]). While the FPU recurrence was investigated in detail for the regimes (i) [4 $-\underline{8}$, , 11] $]$ and (ii) [9], it was not studied for the regime (iii), which case in-

*Electronic address: ruban@itp.ac.ru
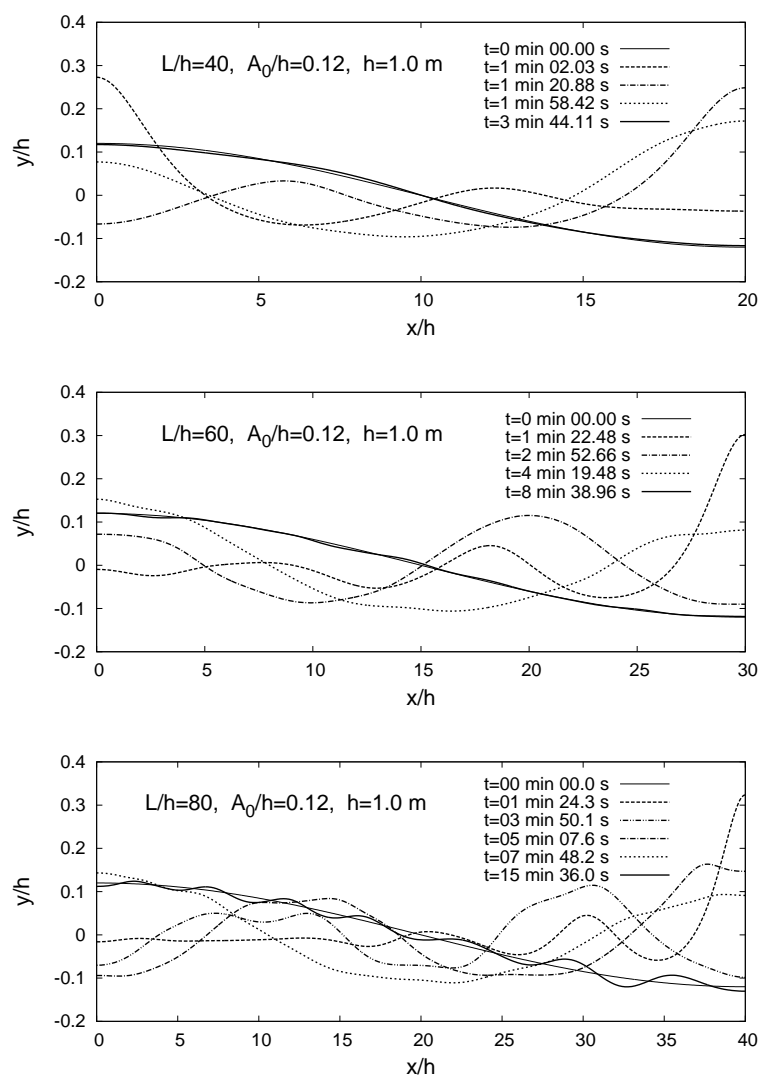

FIG. 1: Three examples of FPU recurrence in water wave systems: the free surface profiles at $t=0$ and at $t=T_{\mathrm{FPU}}(h=$ $\left.1 \mathrm{~m} ; L ; A_{0}=0.12 h\right)$ are very close for $L / h=40$ and for $L / h=$ 60 ; for $L / h=80$ the recurrence is not so perfect but still apparent. The curves with the highest elevation correspond to the moments when a "soliton" collides with the wall.

cludes water waves propagating in a closed flume. The present work is intended to fill this gap by means of direct numerical simulations.

It is important that nonlinear dynamics of free water surface is not exactly integrable (see, e.g., [9, 19], and references therein), and therefore it was not obvious a priori 

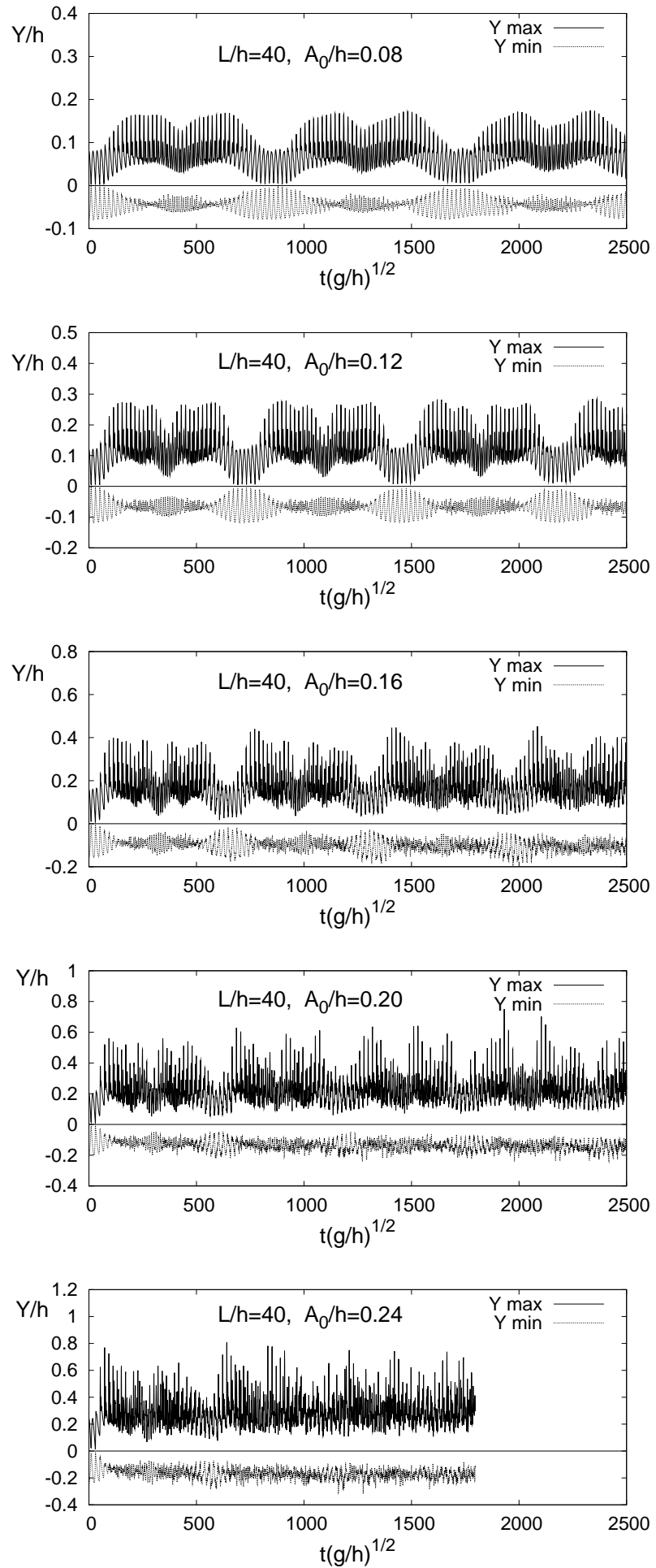

FIG. 2: The maximum and minimum elevations of the free boundary versus time for $L / h=40$ and different initial amplitudes. The peaks of the dependences at approximately regular time intervals correspond to collisions of "solitons" with the "walls".
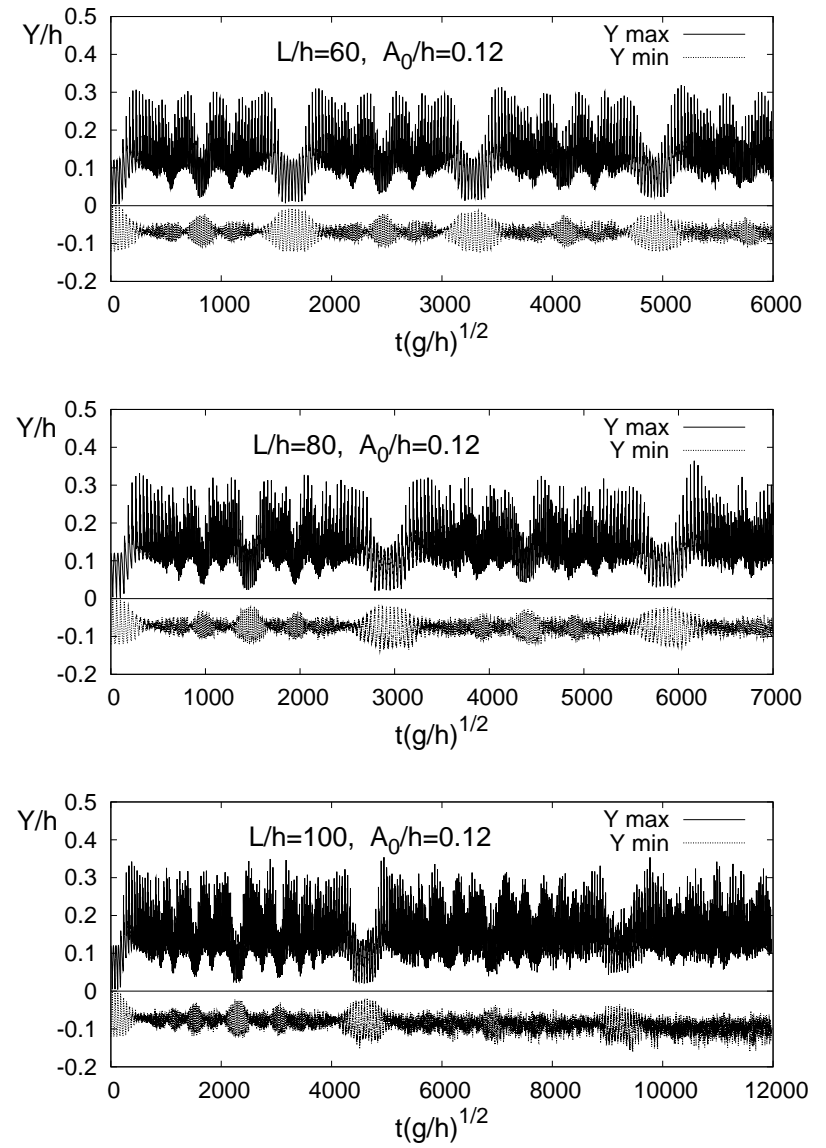

FIG. 3: FPU recurrence for $A_{0} / h=0.12$ and different $L$. With increasing $L$, the dynamics between the recurrence cases becomes more and more complicated: additional minima in the envelope of $Y_{\max }$ appear, which correspond to significant excitation of the second, or the third, or a higher Fourier mode in nearly standing-wave regime.

in what parameter region the FPU recurrence can be observed. To clarify this question, here the highly accurate numerical method based on exact equations of motion for two-dimensional (2D) free-surface potential flows of an ideal incompressible fluid was employed [20, 21], in the most simple variant when the bottom is horizontal at a constant depth $h$. In each numerical experiment, at $t=0$ the free surface profile was taken in the form $y=A_{0} \cos (2 \pi x / L)$ (the gravity acceleration $g$ is directed against $y$ axis), and the velocity field was zero everywhere. Thus, this configuration corresponds to waves in a basin with vertical walls at $x=0$ and $x=L / 2$.

Since the exact equations of motion are written in terms of so called conformal variables, and the surface shape is given in a parametric form $X+i Y=-i h+$ $(1+i \hat{R}) \rho(\vartheta, t)$, with $\hat{R}$ being a linear integral operator [20, 21], it was a nontrivial task how to determine the real function $\rho(\vartheta, 0)$ corresponding to a given initial surface profile $y=\eta_{0}(x)$. This technical problem was solved by 


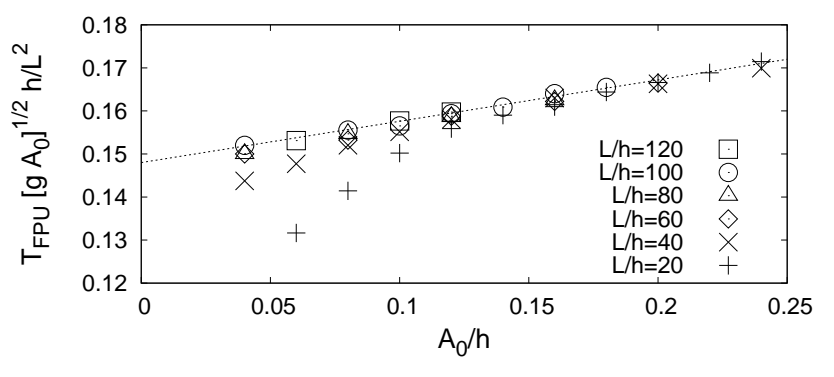

FIG. 4: The time of recurrence, excluding the cases when $\left(A_{0} / h\right)^{1 / 2}(L / h) \lesssim 1$, is well fitted by the formula $T_{\mathrm{FPU}}(g / h)^{1 / 2} \approx C(L / h)^{2}\left(h / A_{0}\right)^{1 / 2}$, with $C=0.148+$ $0.096\left(A_{0} / h\right)$. Roughly, $C=0.16 \pm 0.01$.

an auxiliary numerical procedure, when in one of the two evolutionary equations (namely, in the Bernoulli equation) the combination $\left\{\psi_{t}+g Y\right\}$ was temporarily replaced with the combination $\left\{\psi_{t}+g\left[Y-\eta_{0}(x)\right]+\Gamma \psi\right\}$, where $\psi(\vartheta, t)$ is the surface value of the velocity potential, and $\Gamma=$ const $>0$ is some artificial damping. With this modification, the free surface quickly evolved from $Y=0$ to the required initial profile, and after that we turned to the original equations [20, 21].

The parameters $A_{0}$ and $L$ were taken inside the region $0.04 \leq A_{0} / h \leq 0.24$ and $20 \leq L / h \leq 120$. The computations have demonstrated that the phenomenon of FPU recurrence indeed takes place for moderate initial wave amplitudes $A_{0} \lesssim 0.12 h$. After a few oscillations in a standing-wave regime, the system enters a regime with soliton-like coherent structures moving between the "vertical walls". The shapes of the "solitons" evolve with time, and after a period $T_{\mathrm{FPU}}$, which depends on $h, L$, and $A_{0}$, the system again enters the standingwave regime and approximately repeats the initial state, as it is shown in Fig.1. With a realistic value $h \sim 1 \mathrm{~m}$, the period of recurrence is several minutes.

In Fig.2 and Fig.3, the maximum and minimum elevations of the free boundary versus time are shown for different $A_{0}$ and $L$. These dependences clearly indicate the presence of the FPU phenomenon. However, a "quality" of the recurrence is not uniform in the parametric region: it is better for smaller initial amplitudes and shorter spatial periods $L$. For large amplitudes $A_{0} / h \gtrsim 0.20$, one can hardly recognize more than one recurrence case. In that strongly nonlinear regime, very high waves grow in the system, which can eventually produce sharp crests and break (not shown).
The time of recurrence rapidly increases with the spatial period $L$, and behavior of the system between the recurrences becomes more complicated, since a larger number of solitons participates in the dynamics, $N_{\mathrm{s}} \sim$ $(L / h)\left(A_{0} / h\right)^{1 / 2}$. In the course of evolution, there are some time intervals when a significant part of the energy is concentrated in the second, or in the third, or in a higher Fourier mode.

The numerical results for $T_{\mathrm{FPU}}$ for different $L$ and $A_{0}$ are summarized in Fig.4, which shows that, excluding the cases when $(L / h)\left(A_{0} / h\right)^{1 / 2} \lesssim 1$ (under this condition, the length $L$ becomes too small to contain a soliton with an amplitude about $A_{0}$ ), the time of recurrence is well fitted by a formula

$$
T_{\mathrm{FPU}}\left(\frac{g}{h}\right)^{1 / 2} \approx\left[0.148+0.096 \frac{A_{0}}{h}\right]\left(\frac{L}{h}\right)^{2}\left(\frac{h}{A_{0}}\right)^{1 / 2} .
$$

It should be noted here that in each case the quantity $T_{\mathrm{FPU}}$ was determined rather roughly, just by looking at graphs like those shown in Fig.2 and Fig.3. Therefore the corresponding accuracy is rather low, despite the fact that the accuracy of the simulations is very high (so, the energy conservation was at least 7-8 decimal digits from the beginning of each computation to its end).

In order to observe the FPU recurrence in a real-world flume filled with water, the viscous friction near the bottom and near the side walls should be taken into account. Thus an additional condition arises, $\gamma \cdot T_{\mathrm{FPU}} \ll 1$, where the damping coefficient $\gamma$ can be estimated as

$$
\gamma \lesssim \frac{1}{h} \sqrt{\nu \sqrt{\frac{g}{h}}} \sim h^{-5 / 4}
$$

where $\nu$ is the kinematic viscosity. Since with fixed ratios $A_{0} / h$ and $L / h$ the time of recurrence behaves as $h^{1 / 2}$, the product $\gamma \cdot T_{\mathrm{FPU}}$ behaves as $h^{-3 / 4}$, and therefore it can be indeed small with sufficiently large $h$.

To conclude, in the present work the phenomenon of Fermi-Pasta-Ulam recurrence was observed in the numerical experiments modeling potential one-dimensional water waves in a closed basin. Based on the numerical results, a simple fitting formula for the period of recurrence is suggested.

These investigations were supported by RFBR grant 09-01-00631, by the "Leading Scientific Schools of Russia" grant 6885.2010.2, and by the Program "Fundamental Problems of Nonlinear Dynamics" from the RAS Presidium.
[1] E. Fermi, J. Pasta, and S. Ulam, Studies of Nonlinear Problems, (Los Alamos Scientific Laboratory Report No. LA-1940, Los Alamos, New Mexico, 1955).

[2] N. J. Zabusky and M. D. Kruskal, Phys. Rev. Lett 15,
240 (1965).

[3] V. E. Zakharov, ZhETF 65, 219 (1973).

[4] A. Thyagaraja, Phys. Fluids 22, 2093 (1979).

[5] H. C. Yuen and W.E. Ferguson, Phys. Fluids 21, 1275 
(1978).

[6] E. Infeld, Phys. Rev. Lett. 47, 717 (1981).

[7] H. C. Yuen and B. M. Lake, Advances Appl. Mech. 22, 67 (1982).

[8] N. N. Akhmediev and V.I. Korneev, Theor. Math. Phys. 69, 1089 (1986).

[9] A. R. Osborne, M. Onorato, M. Serio, and L. Bergamasco, Phys. Rev. Lett 81, 3559 (1998).

[10] G. Van Simaeys, Ph. Emplit, and M. Haelterman, Phys. Rev. Lett 87, 033902 (2001).

[11] Q. Zhu, Y. M. Liu, and D. K. P. Yue, J. Fluid Mech. 496, 213 (2003).

[12] N. J. Zabusky, Chaos 15, 015102 (2005).
[13] G. P. Berman and F. M. Izrailev, Chaos 15, 015104 (2005).

[14] D. J. Kaup, Prog. Theor. Phys. 54, 396 (1975).

[15] B. A. Kupershmidt, Commun. Math. Phys. 99, 51 (1985).

[16] A. O. Smirnov, Theor. Math. Phys. 66, 19 (1986).

[17] L. Bogdanov and V.E. Zakharov, Physica D 165, 137 (2002).

[18] J. E. Zhang and Y. Li, Phys. Rev. E 67, 016306 (2003).

[19] W. Craig, P. Guyenne, J. Hammack, D. Henderson, and C. Sulem, Phys. Fluids 18, 057106 (2006).

[20] V. P. Ruban, Phys. Rev. E 70, 066302 (2004).

[21] V.P. Ruban, Phys. Rev. E 77, 037302 (2008). 